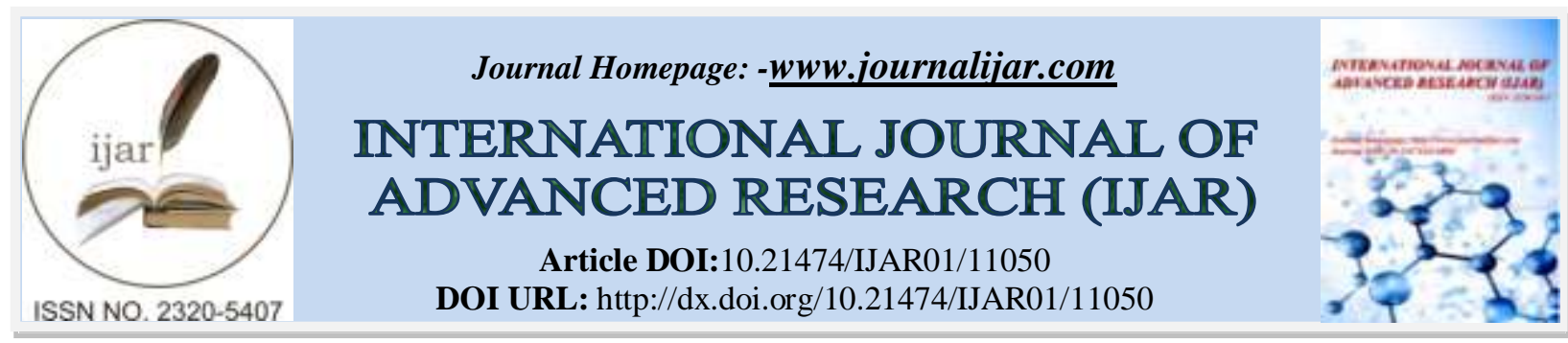

RESEARCH ARTICLE

\title{
DIET THERAPY FOR REFRACTORY EPILEPSY AND ITS RELATIVITY TO DENTISTRY
}

\author{
Dr. Shruti Chopra, Dr. Shveta Sood, Dr. Naresh Sharma and Dr. Akshara Singh \\ Institution of Manav Rachna International Institute of Research and Studies, Department of Pediatric and Preventive
} Dentistry, India.

\begin{abstract}
Manuscript Info
(.........................

Manuscript History

Received: 25 March 2020

Final Accepted: 30 April 2020

Published: May 2020

Abstract

Intractable epilepsy are basically seizures which cannot be controlled with AED one year after onset, even with accurate diagnosis and monitoring of treatment.Intractable epilepsy is linked with an elevated risk of mortality, unemployment and impairment of cognition. Diet therapy measures are in use for the treatment of refractory epilepsy since prehistoric periods. Over the last decade, alternative treatment options, such as diet therapy has gained popularity. Ketogenic diet (KD) is an effective, non-pharmacological treatment for drug resistant epilepsy and difficult to treat epilepsy syndromes of infancy and early epileptic encephalopathies. The diet is deficient in vitamins and minerals and a number of complications have evolved in the form of case reports which mostly reveals oral and systemic complications attributing to a certain deficiency state.
\end{abstract}

Copy Right, IJAR, 2020,. All rights reserved.

\section{Introduction:-}

The word "Epilepsy" is derived from Latin and Greek words for "seizure" or "to seize upon". A seizure can be defined as an alteration of the neurologic function resulting due to an excessive discharge of the neurons in the brain. ${ }^{2}$ Epilepsy is a disease of the brain and is one of the most common chronic neurological illness. ${ }^{3}$ It involves generation of seizures which is associated with a change in behaviour, ability to perceive things, mental activities. It also involves temporary loss of consciousness, and muscular contractions. ${ }^{4}$

"Epilepsy Syndrome" are a cluster of clinical symptoms that occur together in relation with similar seizure(s), age of onset, Electroencephalography (EEG) findings, triggering factors, genetics, natural history, prognosis and response to Antiepileptic drugs (AEDs). ${ }^{2}$

Epilepsy is diagnosed mainly on clinical grounds. According to the International League Against Epilepsy (ILAE), 2014, the diagnosis of epilepsy is made if: -

1. 2 unprovoked seizures occur more than 24 hours apart;

2. 1 unprovoked seizure when there is $\geq 60 \%$ chance of seizure recurrence (similar to that after 2 unprovoked seizures) over the next 10 years; or

3. Epilepsy syndrome is identified. ${ }^{3}$

Epilepsy may be caused due to a variety of factors such as, genetics, developmental disorders, infections, traumatic injuries, neoplasm and diseases of degeneration. ${ }^{5}$ According to the World Health Organisation, approximately 50 
million people worldwide are affected and the incidence is more in developing countries as compared to the developed. ${ }^{6}$

A seizure can be either partial (when epileptical discharges occur in a specific part/region of the brain) or generalised (when discharges affect the entire brain cortex). The diagnosis is made with the help of certain diagnostic aids such as EEG which records the brain waves, and Magnetic Resonance Imaging (MRI). ${ }^{7}$

Treatment is started following diagnosis of epilepsy and AEDs are the main pharmacological treatment indicated. AEDs they suppress (seizures) symptoms of epilepsy and not the disease itself. The AEDs are usually started with a small single dose, which is increased incrementally to the lowest effective maintenance dose. About $50 \%$ of the patients become symptom free, following first AED. ${ }^{3}$

Epidemiological data suggests that recurrent, spontaneous, unprovoked seizures in epilepsy occur in 1 to $3 \%$ of children. Such patients successfully treated with AEDs, however in 10-40\% children, no change is seen in the seizure activity, following drug treatment. ${ }^{5}$

\section{Refractory Epilepsy:}

Seizures that are inadequately controlled even with AEDs, are known as Intractable or refractory. ${ }^{8}$ The ILAE, 2010 defines refractory epilepsy as Inability to obtain seizure control following treatment with two suitable trails of relevantly selected and used AEDs either as a monotherapy or in combination with other drugs. ${ }^{3}$

Intractable epilepsy are basically seizures which cannot be controlled with AED one year after onset, even with accurate diagnosis and monitoring of treatment. ${ }^{5}$ Intractable epilepsy is linked with an elevated risk of mortality, unemployment and impairment ofcognition. ${ }^{9}$

There is no universal criteria over what period of time the seizures must occur in order for it to constitute as refractory. The exact cause of intractable epilepsy is not known but it is established that most patients with refractory epilepsy are resistant to most or all of the AEDs. ${ }^{5}$ However, in a study conducted in apediatric population, identified neuroinfections as a leading cause of resistance to drugs. ${ }^{8}$

A study conducted in North India, identified several predictors of refractory epilepsy such as, structural abnormalities of the cerebrum, resistance to first AED, delay in mile stones, an increase in initial seizure frequency, partial seizure type, febrile seizures and onset before the age of 14 years. ${ }^{8}$

It's linked with excess disability, morbidity and mortality ${ }^{3}$ and affects brain in its early stages of development, spatial learning and memory. ${ }^{5}$

As soon as the diagnosis of drug resistant epilepsy is made on the basis of failure of treatment with 2 AEDs several treatment options are taken into consideration such as surgery. ${ }^{3}$ However, serious complications following temporal lobectomy, callosotomy and hemispherectomy have been reported such as, recurrent bleeding from the Central Nervous System (CNS), post-operative motor deficits, hydrocephalus and wound infections. ${ }^{9}$

Few new AEDs that are identified for the treatment of drug resistant epilepsy (such as lamotrigene, vigbatrin) are tried. Several drug trials on the new agents have shown satisfactory response in adults with refractory epilepsy. Though these agents aremade for use in adults but are being frequently used by clinicians for treating refractory epilepsy in children. ${ }^{9}$

Diet therapy measures are in use for the treatment of refractory epilepsy since prehistoric periods. Over the last decade, alternative treatment options, such as diet therapy has gained popularity. ${ }^{9}$

\section{Ketogenic Diet And Its Relativity To Dentistry:}

Ketogenic diet (KD) is an effective, non-pharmacological treatment for drug resistant epilepsy and difficult to treat epilepsy syndromes of infancy and early epileptic encephalopathies. ${ }^{5}$ It is a high fat diet, developed in the 1990s which is known to initiate certain biochemical changes associated with periods of limited food availability. ${ }^{10}$ 
The favourable outcomes of starvation in treating epilepsy have been noted from $5^{\text {th }}$ century BC. In the early $20^{\text {th }}$ century, it was reported thatimprovement in the frequency of seizures following a period of fasting.In 1921, the effectiveness of KD in treating epilepsy was reported. Research investigators at Johns Hopkins University proposed the antiepileptic effect of KD that causes Ketosis without increased periods of fasting. ${ }^{9}$

Wilder, at Mayo Clinic coined the term Ketogenic diet, a diet which produces high level of ketones in the blood through the consumption of excess fat and less of carbohydrates ${ }^{5}$ and proteins, which forced the body to use ketone bodies (KB) as the main source of fuel. ${ }^{9}$ During the diet, energy consumption of the body transfers from glucose (Primary fuel) to production and utilisation of KB. ${ }^{11}$

K.D was fairly popular in the 1920 s and $1930 \mathrm{~s}{ }^{10}$ but due to the introduction of certain antiepileptic drugs in the late $1930 \mathrm{~s}$ and $1940 \mathrm{~s}{ }^{9}$ the use of diet therapy waned and focus shifted to drug therapy. ${ }^{10}$ However, over the last 15 years there has been a shift in the usage of diet therapies in the treatment of refractory epilepsy. ${ }^{12}$

Diet therapies for the treatment of refractory epilepsy comprises of Classical KD, modified Atkins diet, the Low index treatment diet, Medium chain-triglyceride (MCT) diet, and the Modified MCT. ${ }^{13}$

The classical diet consists of a 4:1 ratio of grams of fat to grams of proteins and carbohydrates combined. Approximately, $90 \%$ of the calories in the diet comes from fat ${ }^{12}$ because per gram of fat 9 calories exist compared to 4 calories/g of carbohydrate or proteins combined. ${ }^{14}$ The calories are confined to $80-90 \%$ of recommended daily allowance initially but may be adjusted gradually. ${ }^{13}$ Children and young adults are started with a ratio of 3:1 of fat to carbohydrate plus protein, as they are rapidly growing. This allows sufficient proteins $(1-1.5 \mathrm{~g} / \mathrm{kg}$ per day) for growth. $^{14}$

The Modified Atkins diet has a similar composition as the classical KD and has a 1:1 ketogenic ratio approximately. ${ }^{15}$ It does not restrict calorie intake and allows unlimited protein and fat intake. The dietary requirements for modifiedatkins diet consists of a concentration of $60-70 \%$ fatty acid, $25-30 \%$ proteins, and approximately $5 \%$ carbohydrates. $^{13}$ The initial daily carbohydrate consumption is estimated to be $10 \mathrm{gms}$ with a gradual increase to $15-20 \mathrm{~g} /$ day after a period of $1-3$ months. ${ }^{15}$ Though, modified atkins diet has several advantages, it is very restrictive for Indians, specially vegetarians. ${ }^{12}$

The Low-glycaemic index(low-GI) diet restricts the carbohydrate intake of the patient. ${ }^{13}$ The low-GI carbohydrates produce comparatively small changes in the blood glucose. It allows intake of total daily carbohydrate of 40$60 \mathrm{~g} /$ day. ${ }^{15}$

Medium chain triglyceride (MCT) diet contains MCT's (decanoic acid and octanoic acid) which has better absorbability and produces more ketones per unity energy during metabolization. Less fat is needed which allows addition of a greater concentration of other substances such as carbs and proteins. The MCT contains 60\% energy which is derived from MCT oil, such high levels of MCT can cause Gastro-intestinal discomfort in children, because of this, Modified MCT diet was introduced. ${ }^{15}$ It consists of $40-50 \%$ long chain fatty acid, $18-20 \%$ protein, $5-10 \%$ carbohydrates and $30 \%$ MCT oil. ${ }^{13}$

K.D should be considered when there is failure in seizure control following treatment with two or three appropriate AEDs. ${ }^{12}$ The diet is initiated in a hospital set up so as to allow careful monitoring of patient's glucose and urinary levels of ketone bodies. It is usually started following fasting ofapproximatelytwenty-four to forty-eighthours (fasting initiation) ${ }^{13}$ or till the presence of ketones is confirmed in the urine. ${ }^{12}$

Patient is provided with $1 / 3^{\text {rd }}$ of the planned calorie intake on the first day of feeding, $2 / 3^{\text {rd }}$ on the second and full caloric intake is allowed on the third day and patient is discharged by the $5^{\text {th }}$ day. ${ }^{12}$

There are several advantages of fasting initiation such as a shorter duration of time is taken for the onset of ketosis, hospital stay allows screening of any underlying metabolic disorders and ketosis can be carefully monitored. ${ }^{12}$

Non fasting initiation is equally effective and is initiated with a 1:1 ration of fat to carbohydrate plus protein by weight, full calorie meals and then daily advancement to a 2:1, 3:1 and finally 4:1 ration. Initiation can be done in the outpatient setting. ${ }^{12}$ 
K.D is safe and generally well tolerated by Indians. ${ }^{12}$ In 2009, a study conducted in AIIMS, New Delhi, India, to assess acceptance of KD in young Indian paediatric patients with intractable epilepsy, revealed that out of 41 children initially included in the study, 14 patients dropped out because the children found the diet to be too restrictive. Though, the Indian diets are cereal based and contain less fat as compared to the Western diet, KD was found to be well tolerated by remaining children. The main cause reported for discontinuation of diet by children was unpalatability. ${ }^{16}$

Problems that occur during incorporation of $\mathrm{KD}$ in Indian population are non-availability of packaged foods, interference by the relatives which results in modification of the original diet plan, large percentage of Indians are vegetarians and religious customs resulting in ban of several foods. ${ }^{17}$

Table 1.1:- Sample Diet For Vegetarians.

Non-starchy vegetables: spinach, brocolli, mushrooms, bell peppers.

Healthy fats: olive oil, coconut oil, mct oil.

Coconut products: full-fat coconut milk, coconut scream, unsweetened coconut.

Nut and seed butter: almonds, walnuts, cashew butter.

Berries: blueberries, blackberries and strawberries.

Protein: tofu, nutritional yeast

Herbs and seasonings: basil, salt, pepper, paprika, turmeric.

The exact mechanism of action by which K.D suppresses epilepsy is not known ${ }^{12}$ however, several theories have been put forward that suggest that there is a direct stabilising effect of ketone bodies on the Central Nervous System, acidosis that occurs following ketosis have been shown to modify seizure threshold, changes in the fluid and electrolyte balance and change in lipid concentration induced by diet, has an antiseizure effect. ${ }^{9}$

Acetone and acetoacetate both have shown to have anticonvulsant properties in animal models. ${ }^{12}$ Incomplete oxidation of fat in presence of shortage of carbohydrates, produces ketosis, which has a major role in effectiveness of the diet. ${ }^{14}$

K.D ratios and caloric restrict (CR) are two important factors which help in seizure protection. Higher K.D ratios $(\geq$ 3:1) have shown to increase anticonvulsant efficacy in clinical and experimental models. ${ }^{18}$ K.D has neuroprotective ability i.e., in a state of metabolic stress, K.Bs including $\beta$-hydroxy butyrate serves as an alternative source of energy. A study by Bough et.al. in 2006 revealed that coordinated upregulation of genes present in the hippocampus (which codes for energy metabolism) is seen with K.D. ${ }^{10}$

During the consumption of K.D, neurons resist metabolic stress by serving as a more efficient energy fuel and larger mitochondrial load. This results in an increase in the ability of neurons to withstand metabolic stress that would under normal circumstances lead to cell death and drain the resilience of neurons. ${ }^{10}$

Chronic ketosis modifies Tricarboxylic Acid (TCA) Cycle and increases GABA production, limits regeneration of Reactive Oxygen Species (ROS) and enhances the synthesis of energy in the brain.These changes stabilise the synaptic function and increases the resistance to seizures. ${ }^{5}$

Like other treatment modalities, K.D is also associated with certain complications such as, dehydration and vomiting (seen mainly during the initiation phase), a fall in blood glucose levels may be seen in some children. Constipation is most common side effect reported in the maintenance phase. ${ }^{12}$ Renal stones are reported in $3-7 \%$ of children on K.D. ${ }^{15}$

The diet is deficient in vitamins and minerals ${ }^{12}$ and a number of complications have evolved in the form of case reports which mostly reveals oral and systemic complications attributing to a certain deficiency state. ${ }^{5}$

Scurvy, due to deficiency of Vitamin C, have been reported which results in prolonged bleeding from the gums, changes in the function of platelet with excessive bruising may be seen. A decrease in bone mass, Vitamin D deficiency osteomalacia and a susceptibility to fractures have also been reported. ${ }^{5}$

Children with chronic illnesses such as epilepsy are on long term medications that may contain sugar and are at a higher risk of caries. In such cases, it may be difficult to maintain dental hygiene. ${ }^{5}$ 
A complete or even partial loss of ketosis can impair seizure control. ${ }^{19}$ So, it is imperative to maintain ketotic state by restricting the intake of carbohydrates. ${ }^{5}$

The term "sugar-free" is generally for diabetics and the medicines may contain carbohydrate, which can lead to a failure in ketosis. The carbohydrate content is reported to be in greater concentrations in drug suspension, in case of tablets that are to be chewed it is reported to be in a moderate concentration andlower in capsules that are to be taken intact. $^{13}$

The K.D is a non-cariogenic diet, as all the cariogenic substances are eliminated. It causes a state of ketosis, which limits the generation of ROS (an active biomarker in periodontal disease), which has a short life span and can cause substantial damage to tissues and cellular components. ${ }^{20}$

The safety of the diet in pregnancy needs to be evaluated. "Fetal hydantoin syndrome" is a disorder of various abnormalities that results due to exposure of foetus to teratogenic AEDs such as phenytoin during pregnancy. ${ }^{21}$

However, Louw E et al, in 2016, reported cases of two pregnant women with epilepsy who were treated with K.D as the only treatment modality (monotherapy) or in combination with other treatment options (adjunctive treatment). In both the cases, K.D was found to be an effective therapy for epilepsy in pregnancy and allowed normal growth of the foetus. ${ }^{22}$

The dental team must carefully evaluate and prescribe tablets, capsules or liquid medications that are free of sugar. ${ }^{5}$ The dentist should select, whenever possible, a medication that will not affectketosis. ${ }^{19}$

\section{Conclusion:-}

The treatment of refractory epilepsy proposes a challenge to the physician due to the resistant nature of the disease. It is important to select a treat modality that effectively controls seizure in children and also maintains the quality of life. The dental health team must carefully monitor the disease state, be aware of the adverse reactions resulting due to long term use of AEDs and the current treatment the child is undergoing and provide a treatment the does not affect the treatment in any way. Reported evidence suggests that the diet therapy has a direct or indirect affect on the oral heath due to deficiency of vitamins and minerals.

\section{References:-}

1. World Health Organization, Global Campaign against Epilepsy, Programme for Neurological Diseases, Neuroscience (World Health Organization), International Bureau for Epilepsy, World Health Organization. Department of Mental Health, Substance Abuse, International Bureau of Epilepsy, International League against Epilepsy. Atlas: epilepsy care in the world. World Health Organization; 2005.

2. Stafstrom CE, Carmant L. Seizures and epilepsy: an overview for neuroscientists. Cold Spring Harbor perspectives in medicine. 2015 Jun 1;5(6):a022426.

3. Perucca P, Scheffer IE, Kiley M. The management of epilepsy in children and adults. Medical Journal of Australia. 2018 Mar;208(5):226-33.

4. Mehmet Y, Senem O, Sulun T, Humeyra K. Management of epileptic patients in dentistry. Scientific Research. 2012 Oct 3(1);47-52.

5. Sharma A, Mathur VP. Refractory epilepsy and the ketogenic diet: pathophysiological aspects and possible implications in dental practice. JISPPD. 2011 Jul 1;29(3):188.

6. del Rosario Cruz-Cruz M, Gallardo-Elías J, Paredes-Solís S, Legorreta-Soberanis J, Flores-Moreno M, Andersson N. Factors associated with epilepsy in children in Mexico: A case-control study. Boletin medico del Hospital Infantil de Mexico. 2017 Sep 1;74(5):334-40.

7. Aragon CE, Burneo JG. Understanding the patient with epilepsy and seizures in the dental practice. Journal of the Canadian Dental Association. 2007 Feb 1;73(1).

8. Amudhan S, Gururaj G, Satishchandra P. Epilepsy in India I: Epidemiology and public health. Annals of Indian Academy of Neurology. 2015 Jul;18(3):263.

9. Lefevre F, Aronson N. Ketogenic diet for the treatment of refractory epilepsy in children: a systematic review of efficacy. Pediatrics. 2000 Apr 1;105(4):46-53.

10. Gasior M, Rogawski MA, Hartman AL. Neuroprotective and disease-modifying effects of the ketogenic diet. Behavioural pharmacology. 2006 Sep;17(5-6):431. 
11. Güntner AT, Kompalla JF, Landis H, Theodore SJ, Geidl B, Sievi NA, Kohler M, Pratsinis SE, Gerber PA. Guiding ketogenic diet with breath acetone sensors. Sensors. 2018 Nov;18(11):3655.

12. Sharma S, Jain P. The ketogenic diet and other dietary treatments for refractory epilepsy in children. Annals of Indian Academy of Neurology. 2014 Jul;17(3):253.

13. Misiewicz Runyon A, So TY. The use of ketogenic diet in pediatric patients with epilepsy. ISRN pediatrics. 2012 Aug 28;2012.

14. Kwiterovich Jr PO, Vining EP, Pyzik P, Skolasky Jr R, Freeman JM. Effect of a high-fat ketogenic diet on plasma levels of lipids, lipoproteins, and apolipoproteins in children. Jama. 2003 Aug 20;290(7):912-20.

15. Kossoff EH, Zupec- Kania BA, Amark PE, Ballaban- Gil KR, Christina Bergqvist AG, Blackford R, Buchhalter JR, Caraballo RH, Helen Cross J, Dahlin MG, Donner EJ. Optimal clinical management of children receiving the ketogenic diet: recommendations of the International Ketogenic Diet Study Group. Epilepsia. 2009 Feb;50(2):304-17.

16. Sharma S, Gulati S, Kalra V, Agarwala A, Kabra M. Seizure control and biochemical profile on the ketogenic diet in young children with refractory epilepsy_-Indian experience. Seizure. 2009 Jul 1;18(6):446-9.

17. Nathan JK, Purandare AS, Parekh ZB, Manohar HV. Ketogenic diet in Indian children with uncontrolled epilepsy. Indian pediatrics. 2009 Aug 1;46(8).

18. Bough KJ, Rho JM. Anticonvulsant mechanisms of the ketogenic diet. Epilepsia. 2007 Jan;48(1):43-58.

19. Lebel D, Morin C, Achim N, Laberge M, Carmant L. The carbohydrate and caloric content of concomitant medications for children with epilepsy on the ketogenic diet. Canadian journal of neurological sciences. 2001 Nov;28(4):322-40.

20. Dahiya P, Kamal R, Gupta R, Bhardwaj R, Chaudhary K, Kaur S. Reactive oxygen species in periodontitis. Journal of Indian Society of Periodontology. 2013 Jul;17(4):411. 